\title{
Analysis of Triopoly Game with Isoelastic Demand Function and Heterogeneous Players
}

\author{
Hong-Xing Yao, ${ }^{1,2}$ Lian Shi, ${ }^{2}$ and Hao $\mathrm{Xi}^{2}$ \\ ${ }^{1}$ School of Finance E Economics, Jiangsu University, Zhenjianig 212013, China \\ ${ }^{2}$ Institute of System Engineering, Jiangsu University, Zhenjianig 212013, China \\ Correspondence should be addressed to Hong-Xing Yao, hxyao@ujs.edu.cn
}

Received 24 August 2011; Accepted 28 October 2011

Academic Editor: Baodong Zheng

Copyright (C) 2012 Hong-Xing Yao et al. This is an open access article distributed under the Creative Commons Attribution License, which permits unrestricted use, distribution, and reproduction in any medium, provided the original work is properly cited.

\begin{abstract}
We analyze a triopoly game model with fully heterogeneous players when the demand function is isoelastic. The three players were considered to be bounded rational, adaptive, and naïve. Existing equilibrium points and their locally asymptotic stability conditions are studied. Complexity of the dynamical system is examined by means of numerical simulations, such as period cycles, bifurcation diagrams, strange attractors and sensitive, dependence on initial conditions. This paper extends the result of Tramontana (2010) who considered a heterogeneous duopoly with isoelastic demand function. Comparisons with respect to the heterogeneous triopoly model of Elabbasy et al. (2009) assuming linear demand function are performed.
\end{abstract}

\section{Introduction}

Oligopolistic market is a universal market mechanism, in which a trade is controlled by a small number of firms producing the same or homogeneous products. These firms must consider not only the market demand but also the strategies of their competitors. The most classical and widely used oligopoly model was proposed by Cournot in 1838 [1]. The mathematician Augustin Cournot investigated a duopoly case, where two firms were naïve players. In 1959, Theocharis reconsidered the Cournot oligopoly model assuming a linear demand function and constant marginal costs then he pointed out that an oligopoly system with $n$ players would be only neutrally stable for three players and unstable for four and more players [2]. In [3], Puu pointed out that the Theocharis argument was stated under more general conditions 20 years earlier by palander [4]. After that the similar conclusion was shown by Ahmed and Agiza for a isoelastic demand function and, again, constant marginal costs. They demonstrated that with four players the Cournot equilibrium is neutrally stable and with five and higher becomes unstable [5]. However, it is highly unlikely that all the players are naïve. Therefore, different approaches to firm behavior were proposed. Players were not only naïve but also bounded rational and adaptive. 
Some authors studied dynamics of the duopoly game with homogenous expectations [6-8]. They demonstrated that dynamics of the Cournot oligopoly games may never converge to equilibrium and in the long-term bounded periodic or chaotic behavior may be observed. In [8], delay is introduced in a bounded rationality duopoly game model. The analysis showed that the delay case increases the domain of stability, and firms using delayed bounded rationality have a higher chance of reaching a Nash equilibrium point. Models with heterogeneous players were studied [9-13]. In [9, 10], Agiza and Elsadany investigated dynamics of a duopoly game with bounded rational and naïve (adaptive) assuming linear inverse demand function and linear cost functions. Zhang et al. [11] evidenced chaotic behavior in a modification of the game by Agiza and Elsadany [9] introducing nonlinear, in particular quadratic, instead of linear cost functions and thus increasing marginal costs. Dubiel-Teleszynski [12] amended the game by Agiza and Elsadany [10] replacing linear with nonlinear cost functions. Nonlinear demand function was introduced into the duopoly game of Agiza and Elsadany [9] by Tramontana [13]. In theory, there are another three kinds of duopoly game model. These are bounded rational, and adaptive players facing nonlinear demand function and linear cost functions, bounded rational and naïve players assuming nonlinear demand function and cost functions and bounded rational, and adaptive players facing nonlinear demand function and cost functions.

In oligopoly model, all players maximize their profits while triopoly game is a oligopoly market with three players. The dynamics of a triopoly game with homogenous players were discussed in [14-18]. But, the same with duopoly game, it is impossible that all players have the same expectations. Recently, models with heterogeneous players were studied in $[19,20]$. In these two papers, triopoly with different expectations were used, namely bounded, rational, adaptive, and naïve expectations. The stability of the triopoly game, bifurcation, and chaotic behavior were analyzed. And the difference of the two papers is as follows: one uses linear cost functions; the other applies nonlinear cost functions. We know isoelastic demand function has its advantages. The advantages are that it always results when the consumers maximize utility functions of the Cobb-Douglas type. Then, all the consumers spend constant budget shares on each commodity and they have demand functions of the same type. In this paper, isoelastic demand function is introduced into the triopoly game. And the three players are completely heterogeneous.

This paper is organized as follows. In Section 2, the triopoly game model with isoelastic demand function and fully heterogeneous players is briefly described. The conditions of the existence and local stability of fixed point will be given in Section 3. In Section 4, numerical simulations are used to demonstrate complexity of the system. Finally, the conclusion of the paper is provided in Section 5.

\section{Triopoly Game with Isoelastic Demand Function and Heterogeneous Players}

We consider a market served by three firms, where players produce homogeneous goods which are perfect substitutes. Let $q_{i}(t)$ denote the quantity supplied by $i$ th firm, $i=1,2,3$ during period $t$. We assume an isoelastic demand function that has the form

$$
p=\frac{1}{Q}
$$


which is founded on the hypothesis of the Cobb-Douglas utility function of the agents $[3,6$, 13 , where $Q=\sum_{i=1}^{3} q_{i}$ is the total supply, and the residual supply (not under the control of the $i$ th firm) is $Q_{i}=Q-q_{i}$. The cost function has the linear form

$$
C_{i}\left(q_{i}\right)=c_{i} q_{i}
$$

where $c_{i}$ is the marginal cost of $i$ th firm, so $c_{i}$ is positive constant. The profit of $i$ th firm $i=$ $1,2,3$, is given by

$$
\Pi_{i}=p q_{i}-C_{i}\left(q_{i}\right)=\frac{q_{i}}{Q_{i}+q_{i}}-c_{i} q_{i}
$$

At each period $t$, every player must form an expectation of the rival's output in the next time period in order to determine the corresponding profit-maximizing quantities for period $t+1$. If we denote by $q_{i}(t)$ the output of $i$ th player at time period $t$, its production $q_{i}(t+1)$ for the next time period $t+1$ is decided by solving the optimization problem:

$$
q_{i}=r_{i}\left(q_{j}\right)=\arg \max _{q_{i}}\left[\frac{q_{i}}{Q_{i}+q_{i}}-c_{i} q_{i}\right], \quad i, j=1,2,3, i \neq j
$$

This optimization problem (2.4) has unique solution in the form

$$
q_{i}=\sqrt{\frac{Q_{i}}{c_{i}}}-Q_{i}
$$

In this work, we consider heterogeneous players in the sense that they adopt different mechanisms to decide the output of each time period. We assume the first player is bounded rational player; hence it does not has a complete knowledge of the demand function of the market and builds his output decision on the basis of the expected marginal profit $\partial \prod_{1} / \partial q_{1}$. If the marginal profit is positive (negative), he increases (decreases) his production $q_{i}$ at the next period output. Then the dynamical equation of the first player has the form

$$
q_{1}(t+1)=q_{1}(t)+\alpha q_{1}(t) \frac{\partial \prod_{1}}{\partial q_{1}(t)}=q_{1}(t)+\alpha q_{1}(t)\left(\frac{q_{2}(t)+q_{3}(t)}{\left(q_{1}(t)+q_{2}(t)+q_{3}(t)\right)^{2}}-c_{1}\right),
$$

where $\alpha$ is a positive parameter which represents the speed of adjustment.

We assume the second player is a adaptive player. When player 2 thinks with adaptive expectation, hence he computes his output with weight between last periods' output $q_{2}$ 
and his reaction function $r_{2}\left(q_{1}, q_{3}\right)$. By using above assumptions and using (2.5), hence the dynamical equation of the adaptive player 2 has the form

$$
q_{2}(t+1)=(1-\beta) q_{2}(t)+\beta\left(\sqrt{\frac{q_{1}(t)+q_{3}(t)}{c_{2}}}-\left(q_{1}(t)+q_{3}(t)\right)\right)
$$

where $\beta \in[0,1]$ is the speed of adjustment of adaptive player.

Let player 3 be a naive player. He computes his outputs from using the reaction function in (2.5), and then the dynamical equation of player 3 is given by

$$
q_{3}(t+1)=\sqrt{\frac{q_{1}(t)+q_{2}(t)}{c_{3}}}-\left(q_{1}(t)+q_{2}(t)\right)
$$

By combining (2.6)-(2.8), we have the triopoly game with fully heterogeneous players in the form

$$
\begin{aligned}
& q_{1}(t+1)=q_{1}(t)+\alpha q_{1}(t)\left(\frac{q_{2}(t)+q_{3}(t)}{\left(q_{1}(t)+q_{2}(t)+q_{3}(t)\right)^{2}}-c_{1}\right), \\
& q_{2}(t+1)=(1-\beta) q_{2}(t)+\beta\left(\sqrt{\frac{q_{1}(t)+q_{3}(t)}{c_{2}}}-\left(q_{1}(t)+q_{3}(t)\right)\right), \\
& q_{3}(t+1)=\sqrt{\frac{q_{1}(t)+q_{2}(t)}{c_{3}}}-\left(q_{1}(t)+q_{2}(t)\right) .
\end{aligned}
$$

Therefore, the system describes the dynamical triopoly game with fully heterogeneous players. In the next section, we study the dynamical behaviors of this model using the location of fixed points of the dynamical system; the determination of the parameters sets for given local stable fixed points. We are interested only in nonnegative trajectories; hence the system is not defined in the origin $(0,0,0)$.

\section{Equilibrium Points and Local Stability}

By setting $q_{i}(t+1)=q_{i}(t)=q_{i}$, we get the following nonlinear algebraic system:

$$
\begin{aligned}
& q_{1}\left(\frac{q_{2}+q_{3}}{\left(q_{1}+q_{2}+q_{3}\right)^{2}}-c_{1}\right)=0, \\
& \sqrt{\frac{q_{1}+q_{3}}{c_{2}}}-\left(q_{1}+q_{2}+q_{3}\right)=0, \\
& \sqrt{\frac{q_{1}+q_{2}}{c_{3}}}-\left(q_{1}+q_{2}+q_{3}\right)=0 .
\end{aligned}
$$


It is easy to work out two equilibrium points:

$$
\begin{gathered}
E_{1}=\left(0, \frac{c_{3}}{\left(c_{2}+c_{3}\right)^{2}}, \frac{c_{2}}{\left(c_{2}+c_{3}\right)^{2}}\right), \\
E^{*}=\left(\frac{2\left(c_{2}+c_{3}-c_{1}\right)}{\left(c_{1}+c_{2}+c_{3}\right)^{2}}, \frac{2\left(c_{1}+c_{3}-c_{2}\right)}{\left(c_{1}+c_{2}+c_{3}\right)^{2}}, \frac{2\left(c_{1}+c_{2}-c_{3}\right)}{\left(c_{1}+c_{2}+c_{3}\right)^{2}}\right),
\end{gathered}
$$

where $E_{1}$ is the boundary equilibrium point and $E^{*}$ is the Nash equilibrium point provided that $q_{1}{ }^{*}>0, q_{2}{ }^{*}>0$ and $q_{3}{ }^{*}>0$; that is,

$$
\begin{aligned}
& c_{2}+c_{3}>c_{1}, \\
& c_{1}+c_{3}>c_{2}, \\
& c_{1}+c_{2}>c_{3} .
\end{aligned}
$$

In mathematics, that is to say, $c_{1}, c_{2}$ and $c_{3}$ must be able to constitute a triangle. Otherwise, there will be one or two firms out of the market.

In order to investigate the local stability of the equilibrium points of $E_{1}$ and $E^{*}$, we return to the three dimensional system. Let $J$ be the Jacobian matrix of the system corresponding to the state variables $\left(q_{1}, q_{2}, q_{3}\right)$, then

$$
\begin{aligned}
& J\left(q_{1}, q_{2}, q_{3}\right) \\
& =\left[\begin{array}{ccc}
1-\alpha c_{1}+\frac{\alpha\left(q_{2}+q_{3}\right)\left(q_{2}+q_{3}-q_{1}\right)}{\left(q_{1}+q_{2}+q_{3}\right)^{2}} & \frac{\alpha q_{1}\left(q_{1}-q_{2}-q_{3}\right)}{\left(q_{1}+q_{2}+q_{3}\right)^{3}} & \frac{\alpha q_{1}\left(q_{1}-q_{2}-q_{3}\right)}{\left(q_{1}+q_{2}+q_{3}\right)^{3}} \\
\beta\left(\frac{1}{2 \sqrt{c_{2}\left(q_{1}+q_{3}\right)}}-1\right) & 1-\beta & \beta\left(\frac{1}{2 \sqrt{c_{2}\left(q_{1}+q_{3}\right)}}-1\right) \\
\frac{1}{2 \sqrt{c_{3}\left(q_{1}+q_{2}\right)}}-1 & \frac{1}{2 \sqrt{c_{3}\left(q_{1}+q_{2}\right)}}-1 & 0
\end{array}\right] .
\end{aligned}
$$

The stability of equilibrium points will be determined by the nature of the equilibrium eigenvalues of the Jacobian matrix evaluated at the corresponding equilibrium points.

Proposition 3.1. The boundary equilibrium point $E_{1}$ of the system (2.9) is asymptotically stable provided that

$$
\begin{array}{cl}
-2<\alpha\left(c_{2}+c_{3}-c_{1}\right)<0 & \text { if }(3-2 \sqrt{2}) c_{3}<c_{2}<(3+2 \sqrt{2}) c_{3}, \\
-2<\alpha\left(c_{2}+c_{3}-c_{1}\right)<0, \quad \beta<\frac{4 c_{2} c_{3}}{\left(c_{2}-c_{3}\right)^{2}} & \text { if } 0<c_{2} \leq(3-2 \sqrt{2}) c_{3} \cup c_{2} \geq(3+2 \sqrt{2}) c_{3} .
\end{array}
$$


Proof. The Jacobian matrix at $E_{1}$ takes the form:

$$
J\left(E_{1}\right)=J\left(0, q_{2}, q_{3}\right)=\left[\begin{array}{ccc}
1+\alpha\left(c_{2}+c_{3}-c_{1}\right) & 0 & 0 \\
\frac{\beta}{2}\left(\frac{c_{3}}{c_{2}}-1\right) & 1-\beta & \frac{\beta}{2}\left(\frac{c_{3}}{c_{2}}-1\right) \\
\frac{1}{2}\left(\frac{c_{2}}{c_{3}}-1\right) & \frac{1}{2}\left(\frac{c_{2}}{c_{3}}-1\right) & 0
\end{array}\right] .
$$

Its characteristic polynomial can be written as

$$
p(\lambda)=\left(1+\alpha\left(c_{2}+c_{3}-c_{1}\right)-\lambda\right)\left|\begin{array}{cc}
(1-\beta)-\lambda & \frac{\beta}{2}\left(\frac{c_{3}}{c_{2}}-1\right) \\
\frac{1}{2}\left(\frac{c_{2}}{c_{3}}-1\right) & -\lambda
\end{array}\right| .
$$

If we set $\lambda_{1}=1+\alpha\left(c_{2}+c_{3}-c_{1}\right)$ and

$$
r(\lambda)=\left|\begin{array}{cc}
(1-\beta)-\lambda & \frac{\beta}{2}\left(\frac{c_{3}}{c_{2}}-1\right) \\
\frac{1}{2}\left(\frac{c_{2}}{c_{3}}-1\right) & -\lambda
\end{array}\right|=\lambda^{2}-(1-\beta) \lambda+\frac{\beta\left(c_{2}-c_{3}\right)^{2}}{4 c_{2} c_{3}}
$$

then, by Jury stability criterion, we see that the roots of the characteristic polynomial are in the unit disk if the following conditions are fulfilled:
(1) $\left|\lambda_{1}\right|<1$,
(2) $r(1)>0$,
(3) $r(-1)>0$,
(4) $r(0)<1$.

Condition (1) can be written as

$$
-1<1+\alpha\left(c_{2}+c_{3}-c_{1}\right)<1,
$$

which gives us

$$
-2<\alpha\left(c_{2}+c_{3}-c_{1}\right)<0 .
$$

Condition (2) reads as

$$
r(1)=1-(1-\beta)+\frac{\beta\left(c_{2}-c_{3}\right)^{2}}{4 c_{2} c_{3}}=\beta+\frac{\beta\left(c_{2}-c_{3}\right)^{2}}{4 c_{2} c_{3}}>0
$$


which is always fulfilled because $\beta \in[0,1], c_{2}>0, c_{3}>0$. Condition (3) is also always fulfilled because

$$
r(-1)=1+(1-\beta)+\frac{\beta\left(c_{2}-c_{3}\right)^{2}}{4 c_{2} c_{3}}=2-\beta+\frac{\beta\left(c_{2}-c_{3}\right)^{2}}{4 c_{2} c_{3}}>0
$$

which is always fulfilled because $\beta \in[0,1], c_{2}>0, c_{3}>0$. Condition (4) is

$$
r(0)=\frac{\beta\left(c_{2}-c_{3}\right)^{2}}{4 c_{2} c_{3}}<1
$$

Since $\beta \in[0,1]$, so condition (4) is always satisfied if $\left(c_{2}-c_{3}\right)^{2} / 4 c_{2} c_{3}<1$ equivalent to

$$
(3-2 \sqrt{2}) c_{3}<c_{2}<(3+2 \sqrt{2}) c_{3}
$$

while in the case $\left(c_{2}-c_{3}\right)^{2} / 4 c_{2} c_{3} \geq 1$, that is,

$$
0<c_{2} \leq(3-2 \sqrt{2}) c_{3} \cup c_{2} \geq(3+2 \sqrt{2}) c_{3}
$$

the condition (4) is fulfilled if

$$
\beta<\frac{4 c_{2} c_{3}}{\left(c_{2}-c_{3}\right)^{2}}
$$

Inequalities (3.10)-(3.16) prove the result.

In fact, condition (4) indicates a Neimark-Sacker bifurcation. Comparing our boundary equilibrium $E_{1}$ with the boundary equilibrium $E_{1}$ in model of Elabbasy et al. $[19,20]$, their boundary equilibrium is a saddle point, but ours is asymptotically stable under conditions (3.5). This is the consequence of isoelastic demand function and difference between our model and the model of Elabbasy et al. [20].

\subsection{Local Stability Analysis of the Nash Equilibrium Point}

$E^{*}$ is somewhat complex. The coordinates of $E^{*}$ imply that in the Nash equilibrium the output produced by the more efficient firm is higher than the rival's one. The profits corresponding to the Nash equilibrium are

$$
\prod_{1}^{*}=\frac{c_{2}+c_{3}-c_{1}}{c_{1}+c_{2}+c_{3}}, \quad \prod_{2}^{*}=\frac{c_{1}+c_{3}-c_{2}}{c_{1}+c_{2}+c_{3}}, \quad \prod_{3}^{*}=\frac{c_{1}+c_{2}-c_{3}}{c_{1}+c_{2}+c_{3}}
$$

Obviously, the more efficient firm achieves a higher profit. Therefore, in the Nash equilibrium, in order to achieve a higher profit, it is not relevant the mechanism adopted to adjust 
the output but the efficiency of the firm. We promote Tramontana's conclusion in [13] from heterogeneous duopoly to fully heterogeneous triopoly.

Next, we investigate the local stability of the Nash equilibrium. The Jacobian matrix at $E^{*}$ takes the form:

$$
J\left(E^{*}\right)=\left[\begin{array}{ccc}
1-2 \alpha c_{1} \frac{c_{2}+c_{3}-c_{1}}{c_{1}+c_{2}+c_{3}} & \frac{\alpha\left(c_{2}+c_{3}-c_{1}\right)\left(c_{2}+c_{3}-3 c_{1}\right)}{2\left(c_{1}+c_{2}+c_{3}\right)} & \frac{\alpha\left(c_{2}+c_{3}-c_{1}\right)\left(c_{2}+c_{3}-3 c_{1}\right)}{2\left(c_{1}+c_{2}+c_{3}\right)} \\
\beta \frac{c_{1}+c_{3}-3 c_{2}}{4 c_{2}} & 1-\beta & \beta \frac{c_{1}+c_{3}-3 c_{2}}{4 c_{2}} \\
\frac{c_{1}+c_{2}-3 c_{3}}{4 c_{3}} & \frac{c_{1}+c_{2}-3 c_{3}}{4 c_{3}} & 0
\end{array}\right] .
$$

By calculation, we get the characteristic polynomial $p(\lambda)$ of the matrix $J\left(E^{*}\right)$ as follows:

$$
p(\lambda)=\lambda^{3}+A \lambda^{2}+B \lambda+C
$$

where

$$
\begin{aligned}
A= & -\left(1-2 \alpha c_{1} \frac{c_{2}+c_{3}-c_{1}}{c_{1}+c_{2}+c_{3}}\right)-(1-\beta) \\
B= & \left(1-2 \alpha c_{1} \frac{c_{2}+c_{3}-c_{1}}{c_{1}+c_{2}+c_{3}}\right)(1-\beta)-\frac{\alpha\left(c_{2}+c_{3}-c_{1}\right)\left(c_{2}+c_{3}-3 c_{1}\right)}{2\left(c_{1}+c_{2}+c_{3}\right)} \beta \frac{c_{1}+c_{3}-3 c_{2}}{4 c_{2}} \\
& -\frac{\alpha\left(c_{2}+c_{3}-c_{1}\right)\left(c_{2}+c_{3}-3 c_{1}\right)}{2\left(c_{1}+c_{2}+c_{3}\right)} \frac{c_{1}+c_{2}-3 c_{3}}{4 c_{3}}-\beta \frac{c_{1}+c_{3}-3 c_{2}}{4 c_{2}} \frac{c_{1}+c_{2}-3 c_{3}}{4 c_{3}} \\
C= & \left(1-2 \alpha c_{1} \frac{c_{2}+c_{3}-c_{1}}{c_{1}+c_{2}+c_{3}}\right) \beta \frac{c_{1}+c_{3}-3 c_{2}}{4 c_{2}} \frac{c_{1}+c_{2}-3 c_{3}}{4 c_{3}} \\
& -\frac{\alpha\left(c_{2}+c_{3}-c_{1}\right)\left(c_{2}+c_{3}-3 c_{1}\right)}{c_{1}+c_{2}+c_{3}} \beta \frac{c_{1}+c_{3}-3 c_{2}}{4 c_{2}} \frac{c_{1}+c_{2}-3 c_{3}}{4 c_{3}} \\
& +\frac{\alpha\left(c_{2}+c_{3}-c_{1}\right)\left(c_{2}+c_{3}-3 c_{1}\right)}{2\left(c_{1}+c_{2}+c_{3}\right)}(1-\beta) \frac{c_{1}+c_{2}-3 c_{3}}{4 c_{3}}
\end{aligned}
$$

From the Jury stability criterion, the necessary and sufficient conditions for $\left|\lambda_{i}\right|<1, i=1,2,3$ are

$$
\begin{gathered}
1+A+B+C>0 \\
1-A+B-\mathrm{s} C>0 \\
|C|<1 \\
|B-A C|<1-C^{2} .
\end{gathered}
$$

The Nash equilibrium point $E^{*}$ of the system is locally asymptotically stable if the conditions in inequalities (3.21) are all satisfied.

Inequalities (3.21) are very complex. It is too awkward to provide any simplified from. We will give numerical simulations in Section 4.

Under conditions (3.5), $E_{1}$ is asymptotically stable, which means in the long run firm 1 will drop out of the market so that the triopoly evolves into duopoly. However, it is very 


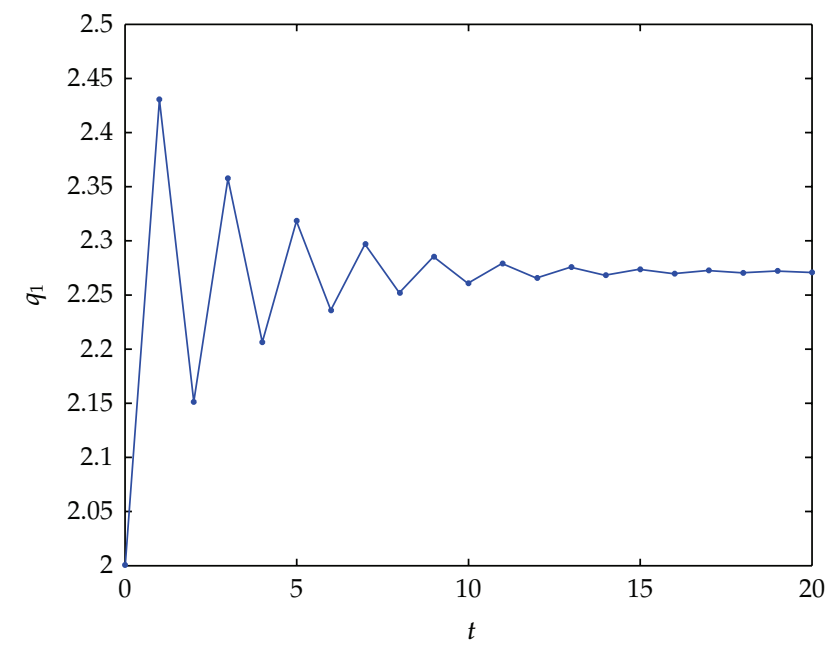

Figure 1: Oscillated convergence to the Nash equilibrium for $\beta=0.2, \alpha=16.0$.

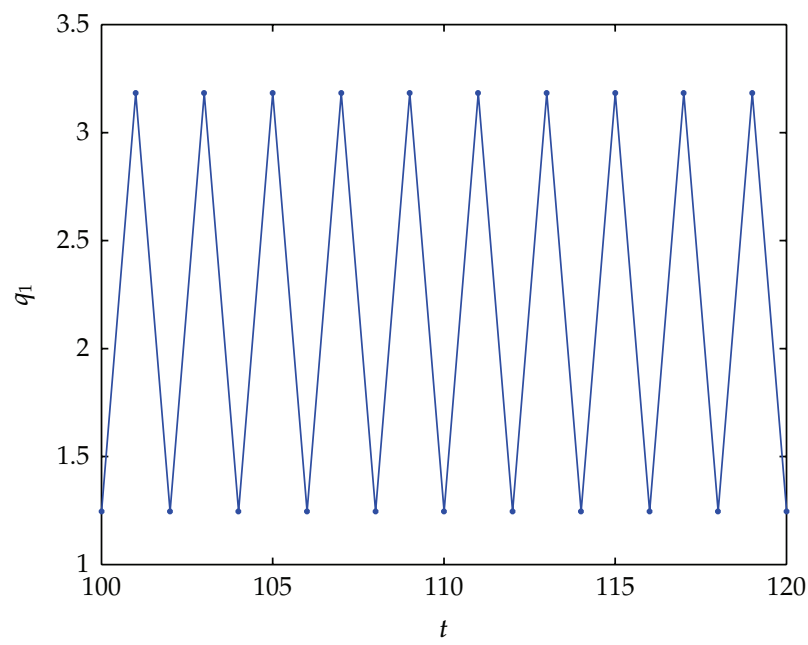

Figure 2: Period-2 cycle for $\beta=0.2, \alpha=19.0$.

interesting that $E^{*}$ will be stable if the parameters satisfy (3.21), since at the Nash equilibrium every firm will stay in the market.

\section{Numerical Simulations}

Only a few of simple dynamic system can be concluded as the dynamic characters by analytical method, so the numerical analysis is the prime method in the research on the complicated dynamical behaviors of the discrete dynamical system (2.9). In addition, we will explore the difference between heterogeneous triopoly game with linear demand function and isoelastic. We show some numerical results such as period cycles, bifurcation diagrams, phase portraits, strange attractors, and sensitive dependence on initial conditions. 


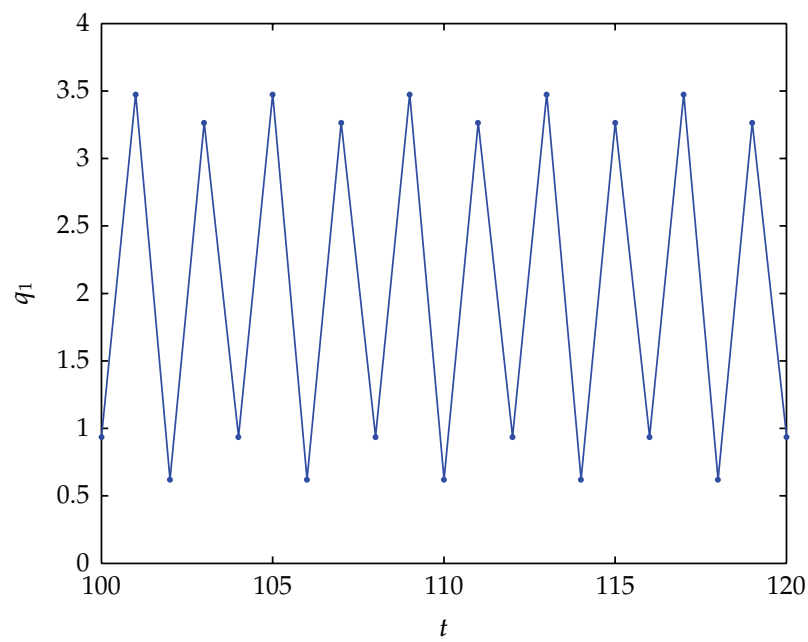

Figure 3: periodic- 4 cycle for $\beta=0.2, \alpha=21.0$.

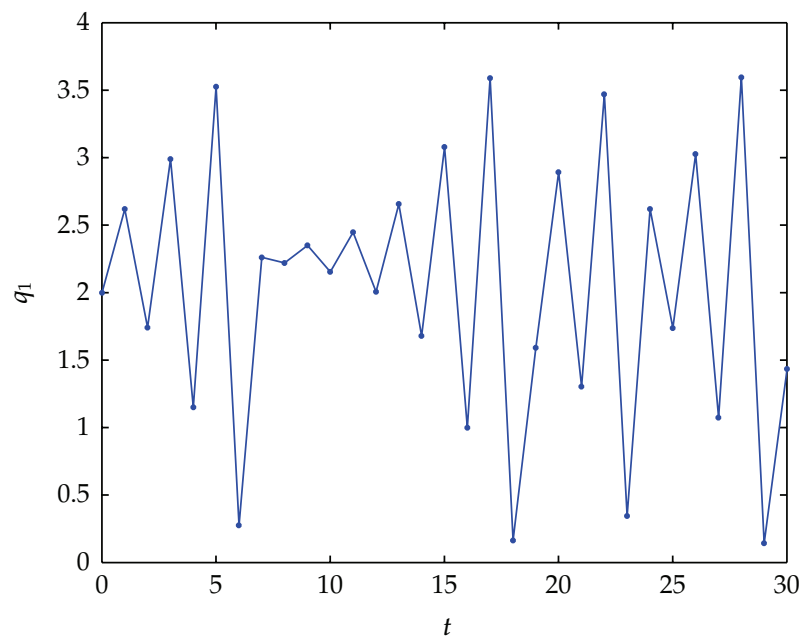

Figure 4: Chaotic values of $q_{1}$ versus time for $\beta=0.2, \alpha=23.1$.

In order to study local stability properties of the Nash equilibrium points, it is convenient to take the model parameters as follows: $q_{1}(0)=2.0, q_{2}(0)=1.5, q_{3}(0)=1.0$ and $c_{1}=0.11, c_{2}=0.15, c_{3}=0.17$ which satisfy (3.3), that is, to say no one firm will drop out the market. Now, the Nash equilibrium point $E^{*}$ is $(2,2715,1.4062,0.9735)$ and

$$
\begin{aligned}
& A=\frac{231 \alpha}{2150}+\beta-2, \\
& B=\frac{427 \alpha \beta}{4000}-\frac{63357 \alpha}{584800}-\frac{53 \beta}{48}+1, \\
& C=-\frac{63 \alpha \beta}{5440}+\frac{21 \alpha}{23392}+\frac{5 \beta}{48}
\end{aligned}
$$




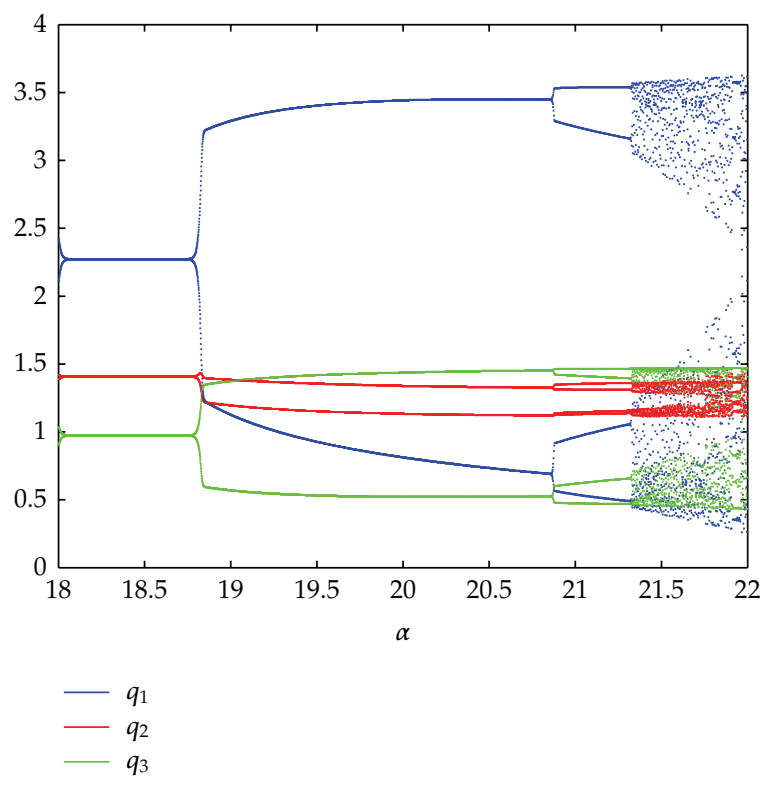

Figure 5: Bifurcation diagram for $\beta=0.4$.

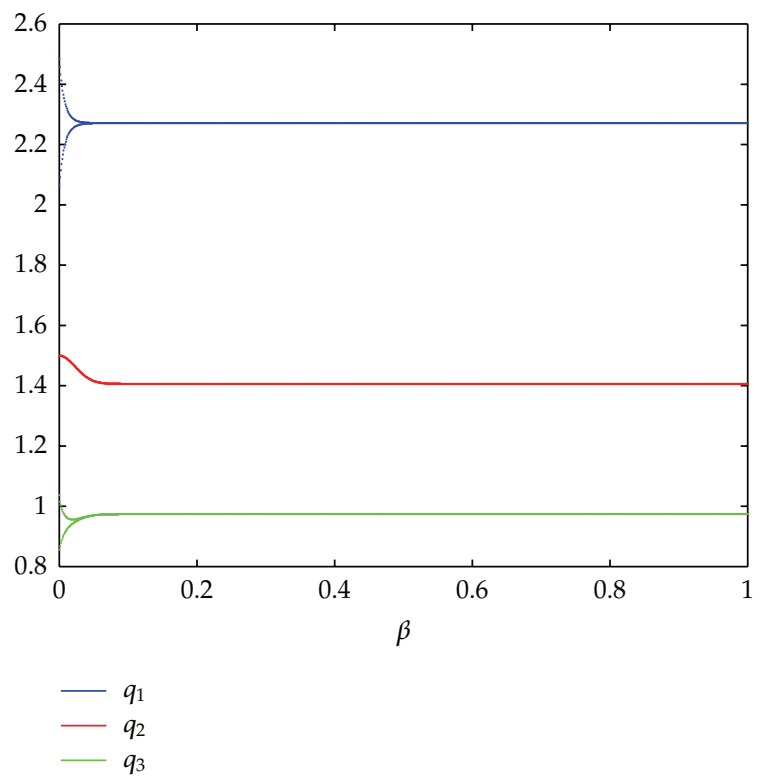

Figure 6: Bifurcation diagram with respect to $\beta$ for $\alpha=18.0$.

Figure 1 shows that the convergence to the Nash equilibrium is oscillating. That is to say, the output of the firm 1 is in some period above and in others below the equilibrium level. Figures 2-4 show the value of the output of the bounded rational player respecting to time. Figure 2 is periodic of period 2; the periodicity of Figure 3 is 4; Figure 4 becomes chaotic. Theoretically, as $\beta=0.2$ and $\alpha>18.44$, the Nash equilibrium point becomes unstable. Period 2 and period 4 bifurcations appear and finally chaotic behaviors occur. Figure 5 also 


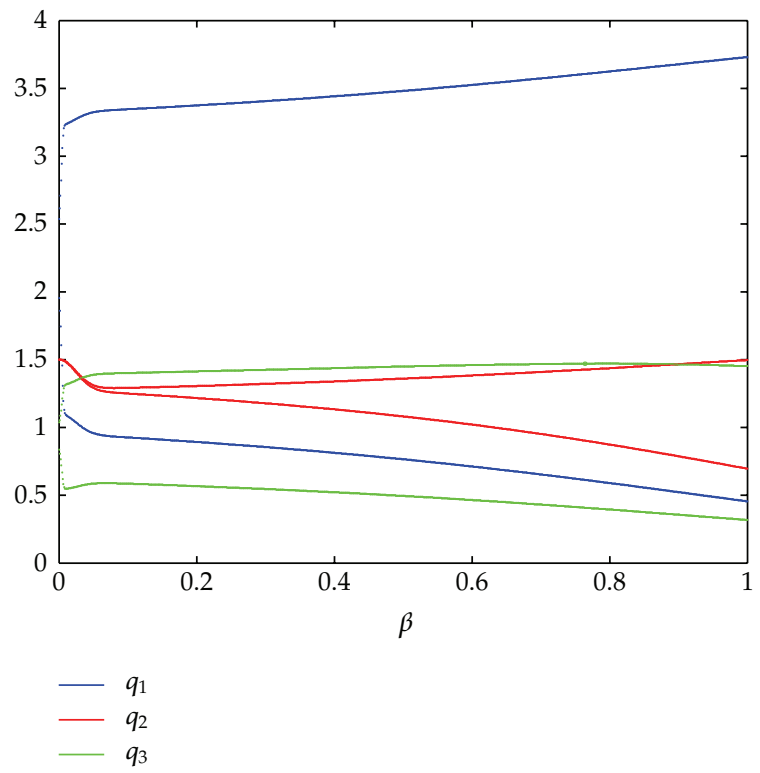

Figure 7: Bifurcation diagram with respect to $\beta$ for $\alpha=20.0$.

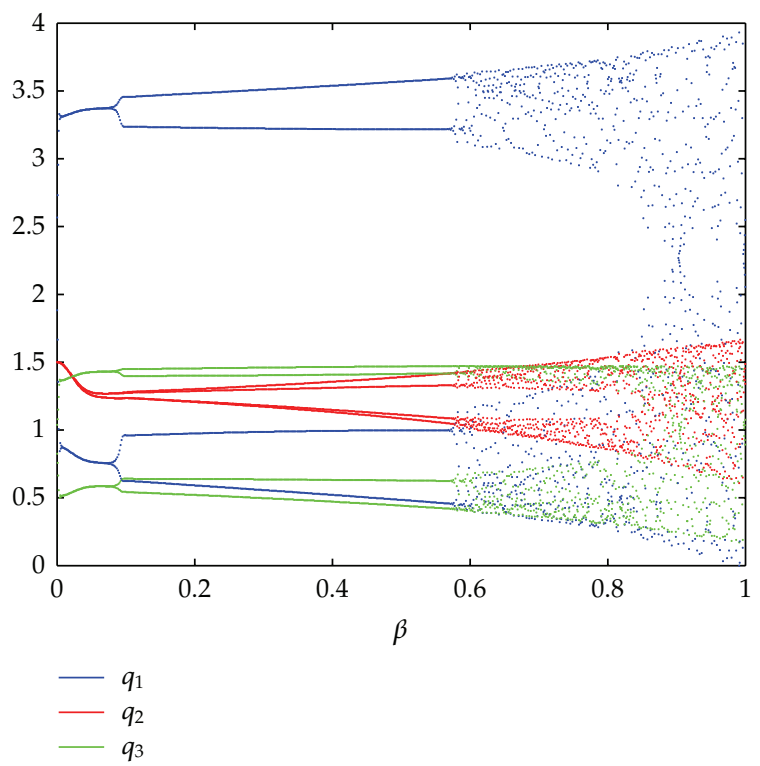

Figure 8: Bifurcation diagram with respect to $\beta$ for $\alpha=21.1$.

shows this kind of evolution direction. Figures 1-4 and Figure 5 are just two ways to show the evolutionary process of system (2.9). From Figures 1-5, everyone can see that the Nash equilibrium point is locally stable for small value of parameter of different $\alpha$, which other authors also concluded. Conversely, a high level of the speed of adjustment of the bounded rational player leads the system to instability. 


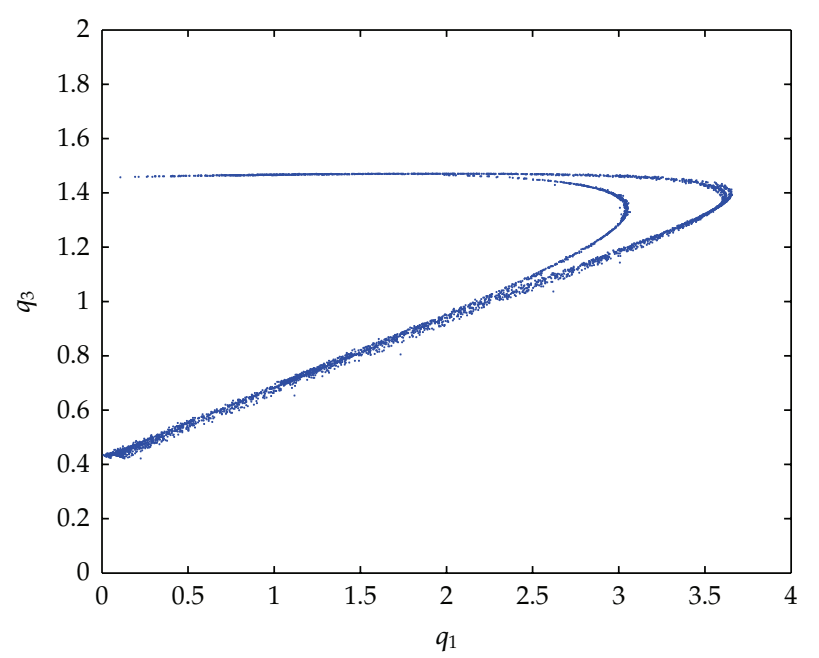

Figure 9: Strange attractor when $\beta=0.2, \alpha=23.1$.

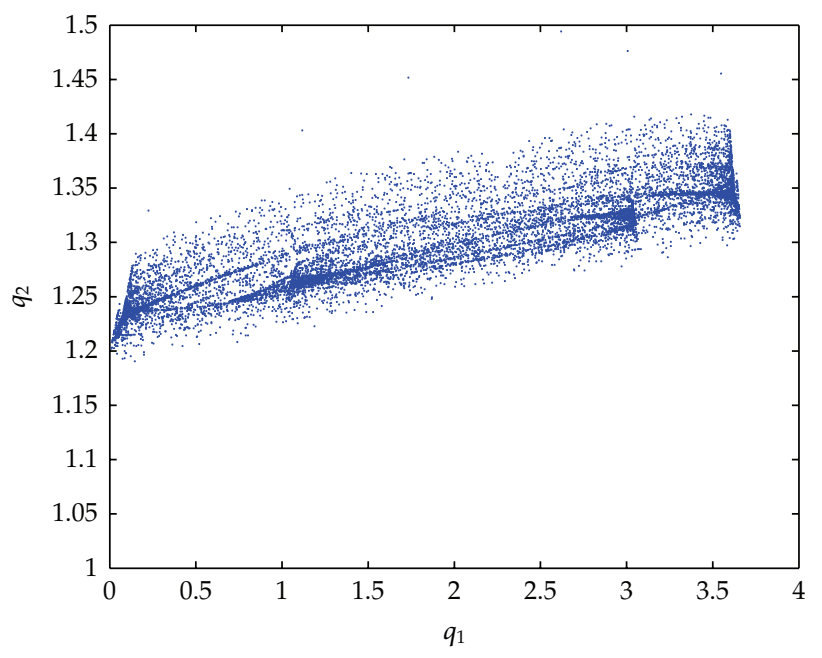

Figure 10: Strange attractor when $\beta=0.2, \alpha=23.1$.

Figures 6, 7 and 8 show the bifurcation diagrams with respect to the parameter $\beta$ and for different $\alpha$. From these figures, we can see that system (2.9) transforms from stable to unstable and chaotic eventually when the value of parameter $\alpha$ increases gradually. That is to say, the market will become more and more confused.

Figures 9, 10, 11 and 12 show the strange attractors for system (2.9). Figures 9-11 show the two-dimension strange attractors. Figure 12 shows the strange attractor from three dimensions.

Using (4.1) and conditions (3.21), we get Figure 13 which shows the stability region of the Nash equilibrium point in the plane $(\alpha, \beta)$. One can see the stability region in this paper is very large compared with respect to the model of Elabbasy et al. [20] and closes to a rectangle region. 


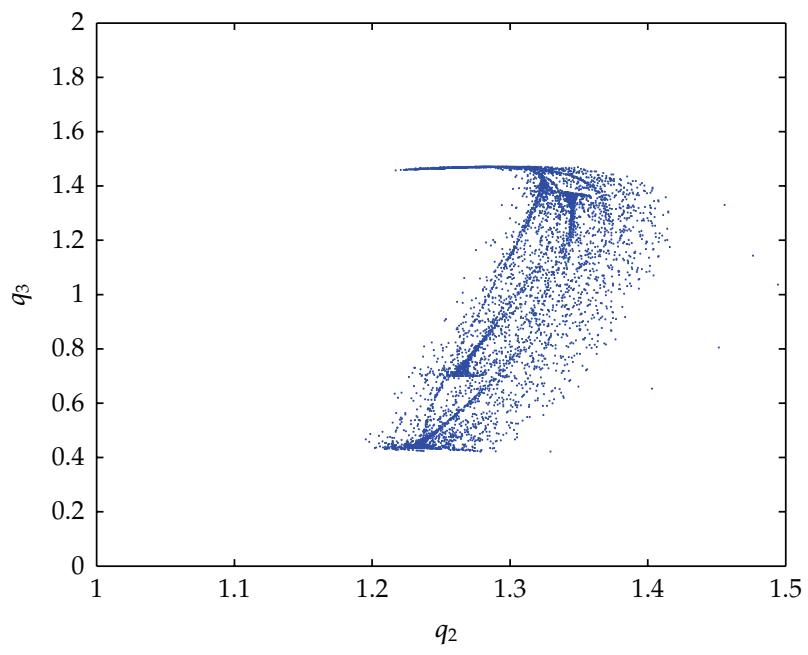

Figure 11: Strange attractor when $\beta=0.2, \alpha=23.1$.

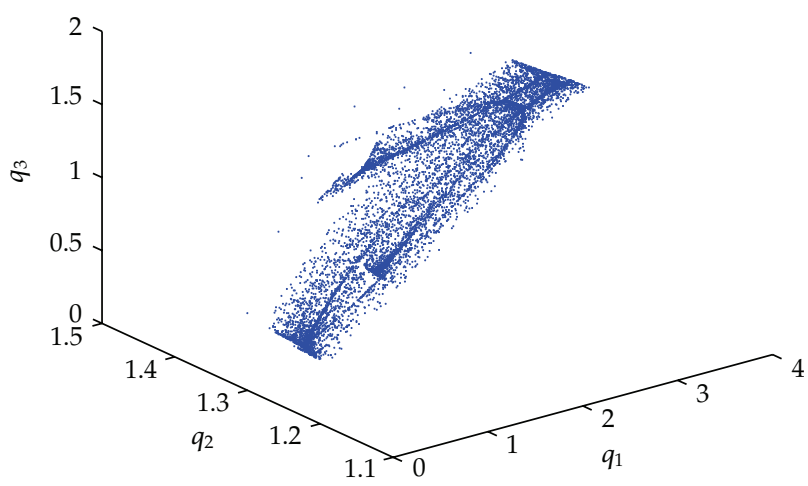

Figure 12: Strange attractor when $\beta=0.2, \alpha=23.1$.

From Figures 1-5 and Figure 13, one can deduce that system (2.9) has similar evolutionary process when $0.4<\beta<1$.

From Figures 6-8 and Figure 13, one can conclude that the Nash equilibrium point is either stable or unstable. That is to say, the speed of adjustment of adaptive player $\beta$ has no effect on stability of the market or not. This constitutes a quite relevant difference between our model and the one proposed by Elabbasy et al. [20].

Sensitive dependence on initial conditions is one of the features of chaotic system. In order to demonstrate sensitive dependence on initial conditions of system (2.9), when $\left(c_{1}, c_{2}, c_{3}, \alpha, \beta\right)=(0.11,0.15,0.17,23.1,0.2)$, we simulated two orbits in Figure 14. The red and blue curves start from the initial points $\left(q_{1}(0), q_{2}(0), q_{3}(0)\right)=(2.0,1.5,1.0)$ and $\left(q_{1}(0), q_{2}(0)\right.$, $\left.q_{3}(0)\right)=(2.0001,1.5,1.0)$, respectively. It shows that, at the beginning, they are indistinguishable, but after a number of iterations, the difference between them builds up rapidly. 


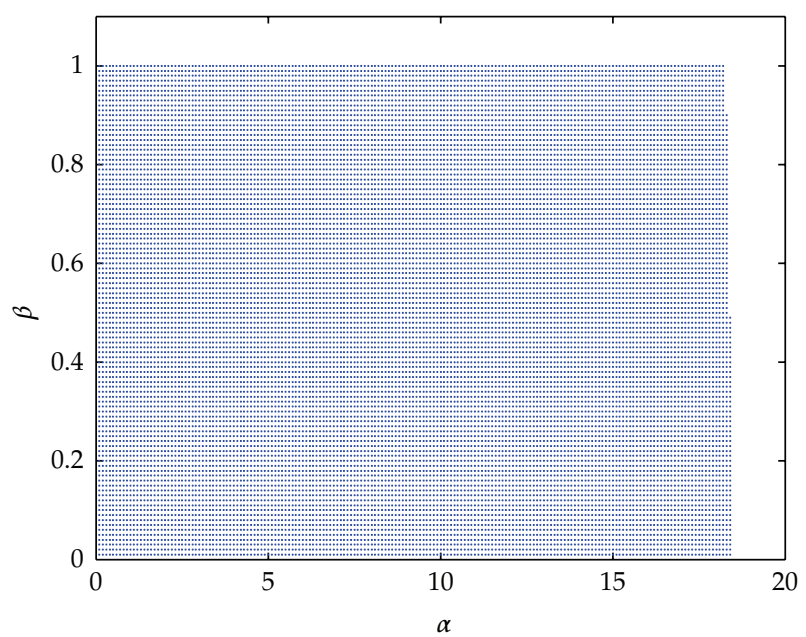

Figure 13: Stability region of the Nash equilibrium point in the plane $(\alpha, \beta)$.

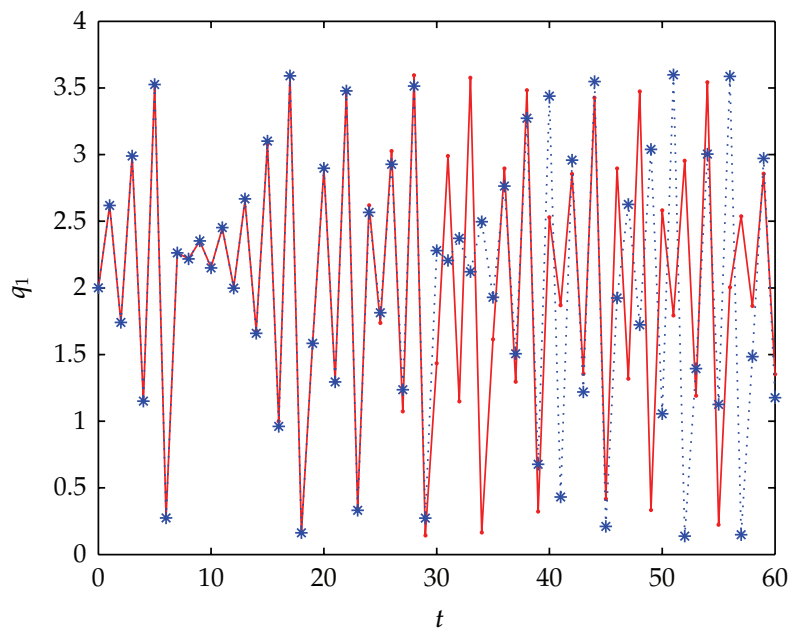

Figure 14: Sensitive dependence on initial conditions for system (2.9).

\section{Conclusion}

In this paper, we propose a fully heterogeneous (bounded rational, adaptive, and naïve expectations) triopoly game model with nonlinear demand function and linear cost functions. The boundary equilibrium point is asymptotically stable under some conditions. The existence condition of the Nash equilibrium point is that the outputs of the three firms must be able to constitute a triangle. Stability region of the Nash equilibrium point is very large compared with respect to heterogeneous triopoly game model with linear demand function and approaches to a rectangle region. The outputs and profits of the firms are not relevant for the decision mechanism the three players adopt in the Nash equilibrium. But the stability of the Nash equilibrium strongly depends on the kind of adjustment adopted. Precisely, it only 
depends on the speed of adjustment of bounded rational player and is not relevant for the adaptive player.

\section{Acknowledgments}

The authors would like to thank the anonymous referees very much for their valuable comments and suggestions. This work was supported by the National Natural Science Foundation of China (no. 70871056) and by the Six Talents Peak Foundation of Jiangsu Province.

\section{References}

[1] A. Cournot, Recherches sur les Principes Mathématiques de la Théoris des Richesses, Hachette, Paris, Farnce, 1838.

[2] R. D. Theocharis, "On the stability of the oligopoly problem," Review of Economic Studies, vol. 27, pp. 133-134, 1959.

[3] T. Puu, "On the stability of Cournot equilibrium when the number of competitors increases," Journal of Economic Behavior and Organization, vol. 66, no. 3-4, pp. 445-456, 2008.

[4] T. Palander, "Konkurrens och marknadsjamvikt vid duopoly och oligopoly," Ekonomisk Tidskrift, vol. 41, no. 124-145, pp. 222-250, 1939.

[5] E. Ahmed and H. N. Agiza, "Dynamics of a cournot game with n-competitors," Chaos, Solitons and Fractals, vol. 9, no. 9, pp. 1513-1517, 1998.

[6] T. Puu, "Chaos in duopoly pricing," Chaos, Solitons and Fractals, vol. 1, no. 6, pp. 573-581, 1991.

[7] M. Kopel, "Simple and complex adjustment dynamics in Cournot duopoly models," Chaos, Solitons and Fractals, vol. 7, no. 12, pp. 2031-2048, 1996.

[8] A. A. Elsadany, "Dynamics of a delayed duopoly game with bounded rationality," Mathematical and Computer Modelling, vol. 52, no. 9-10, pp. 1479-1489, 2010.

[9] H. N. Agiza and A. A. Elsadany, "Nonlinear dynamics in the Cournot duopoly game with heterogeneous players," Physica A, vol. 320, pp. 512-524, 2003.

[10] H. N. Agiza and A. A. Elsadany, "Chaotic dynamics in nonlinear duopoly game with heterogeneous players," Applied Mathematics and Computation, vol. 149, no. 3, pp. 843-860, 2004.

[11] J. Zhang, Q. Da, and Y. Wang, "Analysis of nonlinear duopoly game with heterogeneous players," Economic Modelling, vol. 24, no. 1, pp. 138-148, 2007.

[12] T. Dubiel-Teleszynski, "Nonlinear dynamics in a heterogeneous duopoly game with adjusting players and diseconomies of scale," Communications in Nonlinear Science and Numerical Simulation, vol. 16, no. 1, pp. 296-308, 2011.

[13] F. Tramontana, "Heterogeneous duopoly with isoelastic demand function," Economic Modelling, vol. 27, no. 1, pp. 350-357, 2010.

[14] T. Puu, "Complex dynamics with three oligopolists," Chaos, Solitons and Fractals, vol. 7, no. 12, pp. 2075-2081, 1996

[15] H. N. Agiza, "Explicit stability zones for Cournot game with 3 and 4 competitors," Chaos, Solitons and Fractals, vol. 9, no. 12, pp. 1955-1966, 1998.

[16] H. N. Agiza, G. I. Bischi, and M. Kopel, "Multistability in a dynamic Cournot game with three oligopolists," Mathematics and Computers in Simulation, vol. 51, no. 1-2, pp. 63-90, 1999.

[17] A. Agliari, L. Gardini, and T. Puu, "The dynamics of a triopoly Cournot game," Chaos, Solitons and Fractals, vol. 11, no. 13, pp. 2531-2560, 2000.

[18] T. Puu and M. R. Marín, "The dynamics of a triopoly Cournot game when the competitors operate under capacity constraints," Chaos, Solitons and Fractals, vol. 28, no. 2, pp. 403-413, 2006.

[19] E. M. Elabbasy, H. N. Agiza, A. A. Elsadany, and H. El-Metwally, "The dynamics of triopoly game with heterogeneous players," International Journal of Nonlinear Science, vol. 3, no. 2, pp. 83-90, 2007.

[20] E. M. Elabbasy, H. N. Agiza, and A. A. Elsadany, "Analysis of nonlinear triopoly game with heterogeneous players," Computers and Mathematics with Applications, vol. 57, no. 3, pp. 488-499, 2009. 


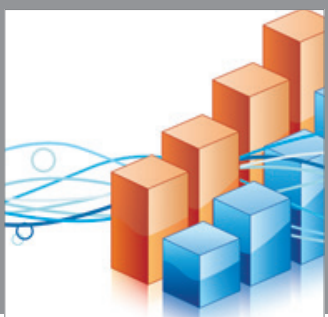

Advances in

Operations Research

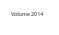

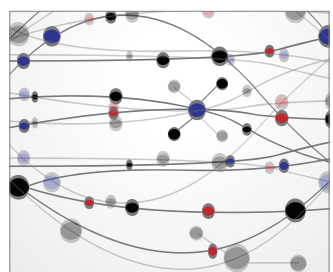

\section{The Scientific} World Journal
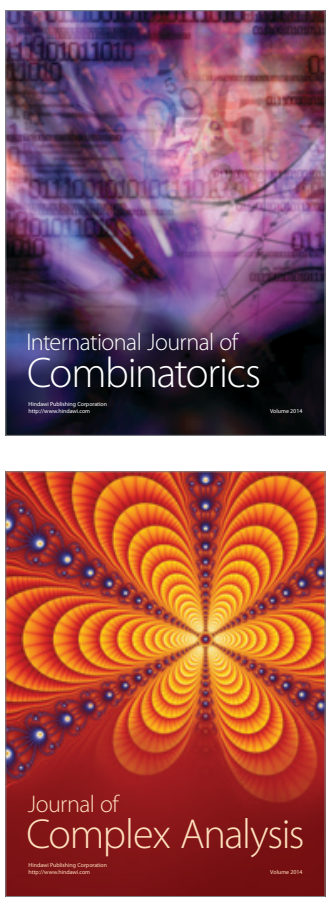

International Journal of

Mathematics and

Mathematical

Sciences
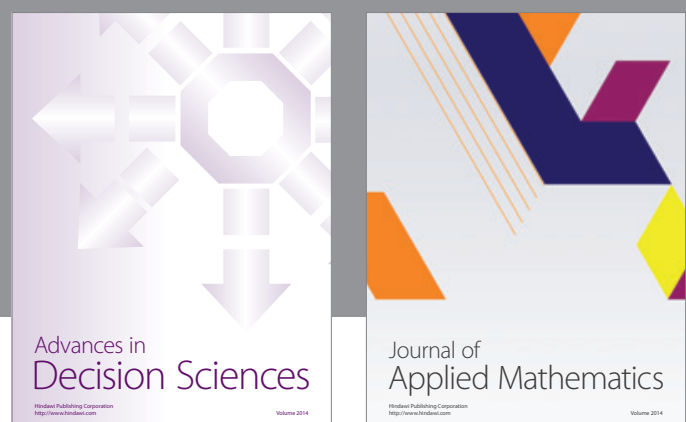

Journal of

Applied Mathematics
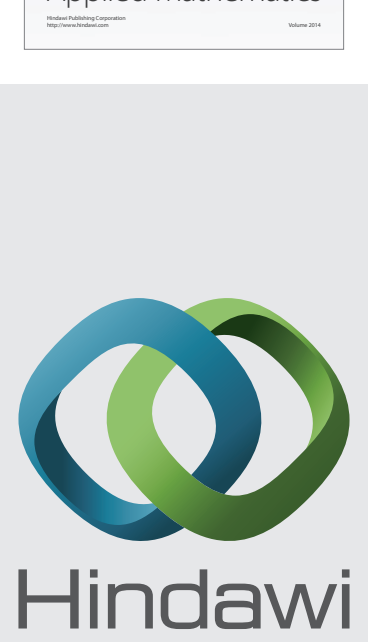

Submit your manuscripts at http://www.hindawi.com
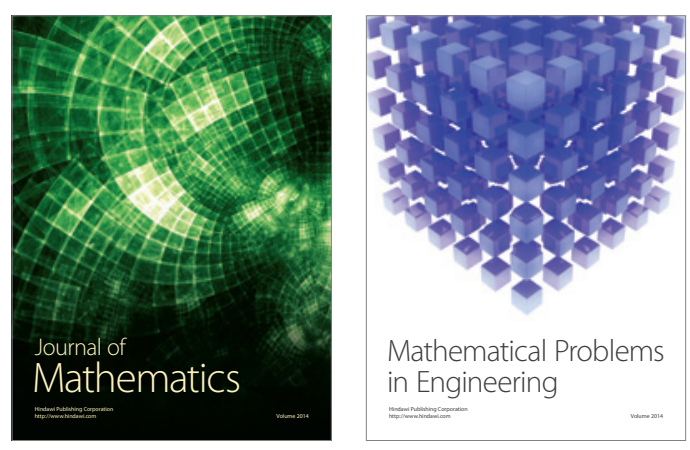

Mathematical Problems in Engineering
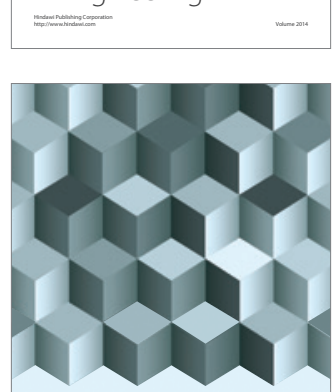

Journal of

Function Spaces
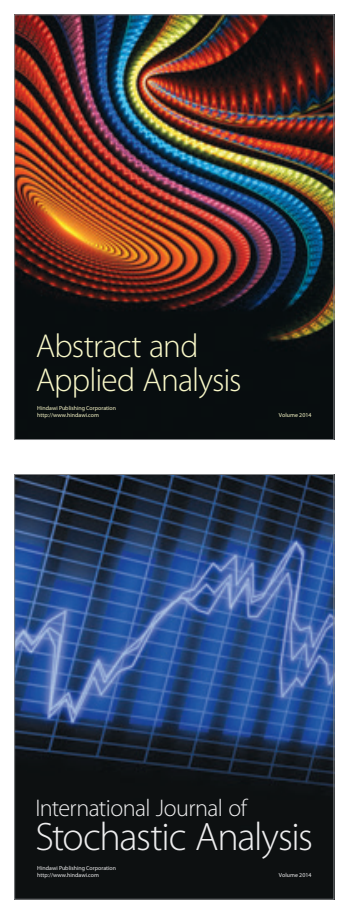

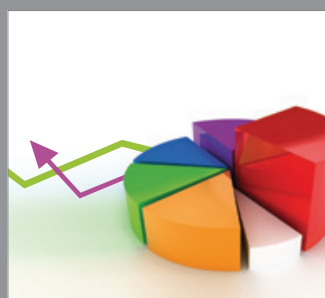

ournal of

Probability and Statistics

Promensencen
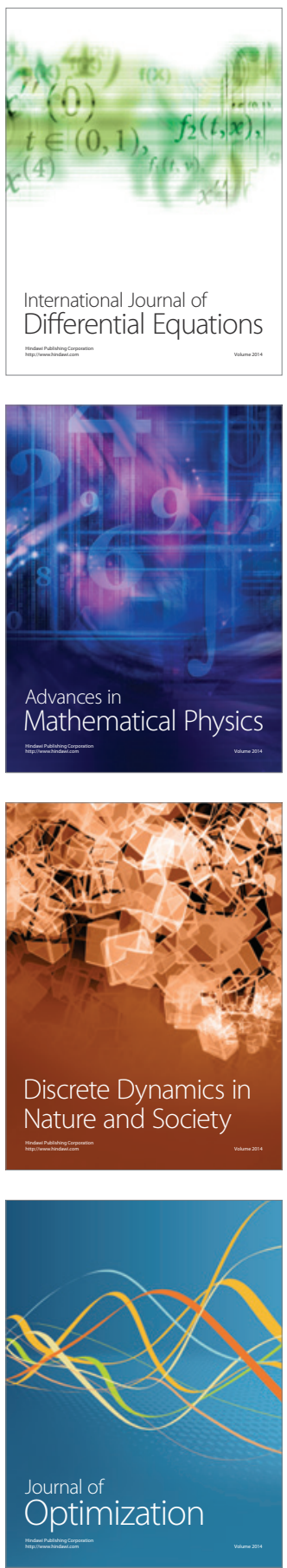